\title{
Esomeprazole inhibits hypoxia/endothelial dysfunction-induced autophagy in preeclampsia
}

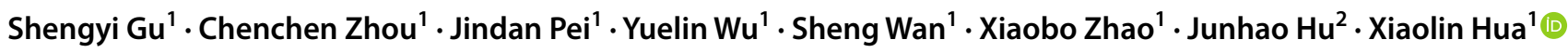

Received: 11 May 2021 / Accepted: 19 January 2022 / Published online: 29 January 2022

(c) The Author(s) 2022

\begin{abstract}
Preeclampsia (PE) affects 3 to 5\% of pregnant women worldwide and is associated with fetal and maternal morbidity and mortality. Although a complete understanding of PE remains elusive, it has been widely accepted that a dysfunction of the placenta plays a key role in the pathogenesis of PE. In this study, we investigated the role of excessive placental autophagy during PE pathogenesis and explored whether esomeprazole ameliorates PE by inhibiting the autophagy in the placenta. The PE cellular model was established by treating the cells' L-NAME and hypoxia. The PE mice model was established by L-NAME administration and was confirmed by the increased systolic blood pressure (SBP) and urinary protein detected. The autophagy and key proteins were detected in human placental tissue, in cells, and in the mice model by Western blot and immunofluorescence staining. Results showed that excessive autophagy could be detected in human PE placental tissue, in the PE cellular model, and in the PE mice model. Hypoxia induces autophagy by activating AMPK $\alpha$ and inhibiting mTOR in vivo and in vitro. Esomeprazole inhibits L-NAME-induced autophagy in mice by inhibiting AMPK $\alpha$ and activating mTOR. In conclusion, this study demonstrates that the excessive autophagy induced by the SIRT1/AMPK $\alpha-m T O R$ pathway plays a significant role in the pathogenesis of PE. However, esomeprazole treatment inhibits AMPK $\alpha$ but activates mTOR, resulting in the inhibition of autophagy in the placenta and, therefore, mitigates PE symptoms.
\end{abstract}

Keywords Placenta $\cdot$ Preeclampsia $\cdot$ Autophagy $\cdot$ Esomeprazole $\cdot$ AMPK $\alpha$-mTOR

\section{Introduction}

Preeclampsia (PE), characterized by hypertension and proteinuria in mid- or late-term pregnancy, is one of the most serious pregnancy complications, causing multiorgan injury (Mol et al. 2016a). Preeclampsia affects 3-5\% of pregnancies worldwide and has been closely associated with fetal and maternal morbidity and mortality (Rana et al. 2019; Mol et al. 2016a).

Shengyi Gu, Chenchen Zhou, and Jindan Pei contributed equally to this work.

Xiaolin Hua

xiaolin_hua@tongji.edu.cn

1 Shanghai First Maternity and Infant Hospital, School of Medicine, Tongji University, Shanghai 200092, China

2 Interdisciplinary Research Center On Biology and Chemistry, Shanghai Institute of Organic Chemistry, Chinese Academy of Sciences, Shanghai 201203, China
An initial asymptomatic phase during the first trimester of gestation is widely accepted as the pathogenesis of preeclampsia, which is characterized by a deficient trophoblast invasion and spiral artery recasting disorder (Phipps et al. 2019), followed by anoxia and malnutrition of the placenta, resulting in hypertension and proteinuria. Moreover, the maternal-fetal interface shows inflammatory overactivation and endothelial dysfunction. The placenta plays a vital role in the pathogenesis of preeclampsia.

Hypoxia, undernutrition, inflammatory overactivation, and endothelial dysfunction can induce autophagy (Sasaki et al. 2007; Hiby et al. 2010). Autophagy is an intracellular self-degrading system characterized by a widespread degradation process for long-lived proteins or cytoplasmic components during undernutrition (Nakashima et al. 2017; Glick et al. 2010). Autophagy's regulatory mechanism is complex, and its upstream signaling pathway mainly involves an mTOR-dependent pathway and an mTORindependent pathway (AMPK, PI3K, Ras-MAPK, p53, PTEN, endoplasmic reticulum stress). Cells use macroautophagy/autophagy to facilitate survival by maintaining 
cellular integrity when experiencing strong environmental stimuli such as hypoxia (Mazure and Pouysségur 2010). In the case of hypoxia, HIF- $1 \alpha$ and HIF- $2 \alpha$ are the mediators of the hypoxic stress signal. The HIF-dependent induction of autophagy by hypoxia has been reported. Upon a hypoxia-mediated autophagy induction, the HIF- $1 \alpha-$ eliminated cells showed a decrease in the levels of the well-known autophagy markers beclin-1 and LC3B-II (Ravanan et al. 2017). HIF activates autophagy through BNIP3 (Bcl-2/E1B $19 \mathrm{kDa}$-interacting protein 3). The $\mathrm{HIF} \alpha$ and IKK mediate the inflammatory and hypoxic signals, activating a cascade of signaling them downstream to activate inflammation and autophagy as stress responses (Bellot et al. 2009). The NF- $\mathrm{KB}$ links autophagy and inflammation via HIF $\alpha$. The mTOR inhibition activates the NF- $\mathrm{KB}$ in the inflammatory pathway, while the mTOR activation initiates the transcriptional activity of HIF $\alpha$. Additionally, autophagy is necessary for embryonic development and placenta implantation in mammals (Boya et al. 2018; Nakashima et al. 2019). For placental villi bathed in maternal blood, once the placenta is in an autophagy disorder, abundant toxic factors, such as soluble Fms-like tyrosine kinase-1(sFlt-1), are released and damage maternal vasculature (James et al. 2005). Thus, researchers have devoted increasing attention to the role of autophagy in the placenta.

The termination of the pregnancy is the first choice for the treatment of preeclampsia, which potentially generates iatrogenic prematurity in babies and affects neonatal outcomes (Tomimatsu et al. 2019). To prolong pregnancy, there is no treatment for the pathogenesis except for symptomatic treatment. Esomeprazole, a proton-pump inhibitor, is used to treat reflux esophagitis and hyperemesis gravidarum (Malfertheiner et al. 2017). It has been found to significantly reduce the levels of sFlt- 1 and sENG and to effectively prolong pregnancy duration (Onda et al. 2017). Esomeprazole also mitigates endothelial dysfunction by inhibiting the expression of tumor necrosis factor $\alpha$, vascular cell adhesion molecule 1, and endothelin 1 (Sandrim et al. 2019). A randomized placebo-controlled trial found that esomeprazole could prolong gestation three more days compared to the placebo in pregnancies with preterm preeclampsia (Cluver et al. 2018). Therefore, it has been suggested as a potential drug for treating preeclampsia. However, the mechanisms of esomeprazole in placental function remain unclear.

In this study, we report that normal autophagy was necessary for placental function. We investigate the effect of excessive autophagy and the effect of esomeprazole on PE. Finally, we find that esomeprazole inhibits hypoxia/ endothelial dysfunction-induced autophagy via the AMPK/mTOR pathway.

\section{Materials and methods}

\section{Placental tissue collection}

Human placental tissue was collected under appropriate Human Research and Ethics Committee approvals (KS1957). Written and informed consents were obtained from each patient before surgery.

One cubic centimeter of placental tissue was removed from the maternal side of the placenta $(3 \mathrm{~cm}$ from the edge in the 3, 6, 9, and 12 o' clock directions). The PE group was selected based on relevant recommendations by the Chinese Medical Association. The control placental tissues were obtained from age-matched preterm pregnancies with normally developing fetuses that did not have signs of hypertension disorder or other pregnancy-related diseases.

\section{Cell line cultures}

Human chorionic trophoblast cells (HTR-8/Svneo) seeded at a density of $2 * 10^{6}$ cells into $10-\mathrm{cm}$ dishes were kept at $37^{\circ} \mathrm{C}$ in $5 \% \mathrm{CO}_{2}$ and $20 \% \mathrm{O}_{2}$ and cultured in 1640 media (Gibco) with a $10 \%$ Australian fetal bovine serum.

Human umbilical vein endothelial cells (HUVECs) seeded at a density of $2 \times 10^{6}$ cells into $10-\mathrm{cm}$ dishes were kept at $37{ }^{\circ} \mathrm{C}$ in $5 \% \mathrm{CO}_{2}$ and $20 \% \mathrm{O}_{2}$ and cultured in ECM media (Sciencell) with a 5\% Australian fetal bovine serum and EGF.

When performing hypoxia, L-NAME (MCE, HY-18729), and esomeprazole (Sigma-Aldrich, PHR-1585) treatments, HTR-8/Svneo and HUVEC cells were treated with and without either L-NAME $(70 \mathrm{mM})$ or esomeprazole $(50 \mathrm{mM})$ and hypoxia for $48 \mathrm{~h}$.

All cell experiments were repeated three times.

\section{PE mice model}

Eight-week-old female C57BL/6 mice (weighing 25-30 g) were used in this study. Male and female mice were mated in the same cage at a male-to-female ratio of 1:2. The time at which the vaginal plug was found was defined as GD0.5. Subsequently, pregnant mice were divided into three groups: the control group (normal pregnancy, $n=5$ ), PE group $(n=5)$, and treated-PE group (esomeprazole $40 \mathrm{mg} /$ $\mathrm{kg}, n=5)$. The PE and treated-PE groups were treated by continuous treatment with L-NAME dissolved in drinking water ( $1 \mathrm{~g} / \mathrm{L} 10 \mathrm{~mL} /$ day; Sigma) beginning from GD7.5 to GD18.5. From GD7.5 to GD18.5, esomeprazole dissolved in $100 \mu \mathrm{l} 1 \% \mathrm{DMSO}(v / v)$ was injected intraperitoneally for the treated-PE group every day, and an equal volume of $1 \%$ DMSO $(v / v)$ was injected intraperitoneally for the PE and 
control groups each day. At GD20, the mice were sacrificed, and the placenta and blood specimens were collected. All experimental protocols were approved by the ethics committee at Xinhua Hospital, Shanghai Jiaotong University School of Medicine.

\section{Assessment of systolic blood pressure}

The systolic blood pressure (SBP) of each mouse was measured noninvasively at GD18.5 via the Mouse and Rat Tail Cuff Blood Tail-Cuff Device (American IITC). Before each measurement was taken, each mouse was exposed to $37^{\circ} \mathrm{C}$ for $5 \mathrm{~min}$; measurements were taken three times for averaging purposes.

\section{Placental histology}

Placenta specimens from the separate groups were preserved in tissue fixatives, embedded in paraffin after $24 \mathrm{~h}$, and cut into 5- $\mu \mathrm{m}$ sections. After hematoxylin and eosin staining and Masson trichrome staining, morphological evaluations were performed under light microscopy.

\section{Western blot}

Human tissue from control and PE placenta and cell lysates from HTR-8/Svneo cells, HUVEC cells, and mouse placentas from the control, PE, and treated-PE groups were detected by Western blotting, as previously described (Gu et al. 2019). Proteins extracted from tissue and cell samples were fractionated on sodium dodecyl sulfate-polyacrylamide gel and electrophoresis gels and transferred to polyvinylidene fluoride membranes. Primary antibodies against $\beta$-actin $(8427 \mathrm{~s}$, rabbit [WB 1:1000]), phospho-AMPK $\alpha$ (2535, rabbit [WB 1:1000] [IF 1:100]), AMPK $\alpha$ (5831, rabbit [WB 1:1000]), PPAR $\gamma$ (2435, rabbit [WB 1:1000]), SIRT1 (2496, rabbit [WB 1:1000]), mTOR (2983, rabbit [WB 1:1000]), phospho-mTOR (5536, rabbit [WB 1:1000]), p53 (2527, rabbit [WB 1:1000] [IF 1:100]), SQSTM1/p62 (7695, rabbit [WB 1:1000] [IF 1:100]), LC3B (3868, rabbit [WB 1:1000] [IF 1:100]), and PI3 kinase class III (4263, rabbit [WB 1:1000]) were purchased from CST. The primary antibodies against HIF- $1 \alpha$ (ab1, mouse [WB 1:1000]) and secondary antibodies were HRP-linked using goat anti-rabbit and goat anti-mouse IgG antibodies (CST, 7074, 7076). The bands were visualized using an ECL kit. Band densitometries in equiconditional area were evaluated by ImageJ. The intensity of both the target protein bands and corresponding internal reference bands was quantified. The intensity value of target protein bands was normalized to the value of the reference bands.

\section{Immunofluorescence staining}

Immunofluorescence staining was performed as previously described. Placenta specimens in distinct groups were preserved in tissue fixatives, embedded in paraffin after $24 \mathrm{~h}$, cut into $5-\mu \mathrm{m}$ sections, and permeabilized with $0.2 \%$ Triton X-100 in PBS. HTR-8/Svneo cells and HUVEC cells were fixed with $4 \%$ PFA for 15 min and permeabilized with $0.2 \%$ Triton X-100 in PBS. Placental sections and cells were precultured in a blocking solution at room temperature to block nonspecific binding sites. They were then cultured with primary antibodies overnight at $4{ }^{\circ} \mathrm{C}$. Placental sections and cells were treated with DAPI (40, 6-diamino2-phenylindole) for nuclear detection. Fluorescence images were observed and captured using confocal microscopy.

\section{Statistics}

The data are represented as mean $\pm \mathrm{SD}$. One-way analysis of variance (ANOVA) was employed for multiple comparisons, followed by Turkey post hoc testing using SPSS 23.0 and GraphPad Prism 9. A $P$ value or adjusted $P$ value less than 0.05 was considered to be statistically significant.

\section{Results and discussion}

\section{Results}

\section{Increased autophagy in placentas of PE patients}

We first histologically analyzed the structure of healthy and PE placentas. H\&E and Trichrome Masson's staining of placenta sections showed that PE placentas showed abnormal structure compared to healthy controls, including reduced branching, compaction of the labyrinth area, increased fibrosis, and reduced vascularization (Fig. 1a-b'). Previous studies have suggested that autophagy may play a role in PE pathogenesis; therefore, we analyzed the LC3B, a well-characterized marker of autophagy, and protein levels in placentas using the Western blot. Results showed that the LC3B expression was significantly increased in the PE placentas than in the control group (Fig. $1 \mathrm{c}, \mathrm{c}^{\prime}, P<0.05$ ). The immunofluorescence-staining analysis of $\mathrm{PE}$ and healthy placentas further confirmed the elevated expression of LC3B in PE placentas (Fig. 1d-e", $P<0.05$ ).

\section{Hypoxia induces autophagy by activating AMPKa and inhibiting mTOR in PE human placenta}

Interestingly, Western blot analysis showed that the HIF-1 $\alpha$ protein level was significantly increased in PE placenta 

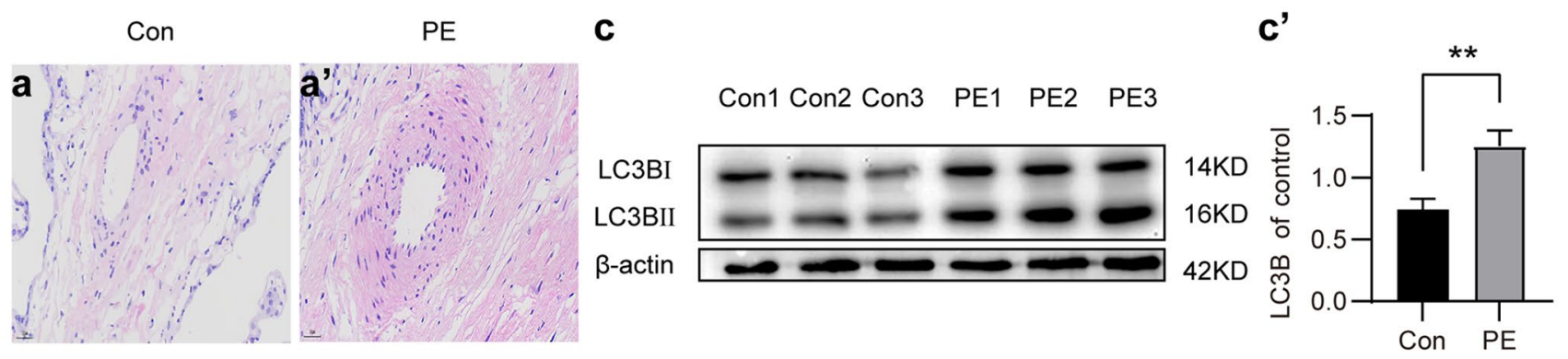

Con

PE

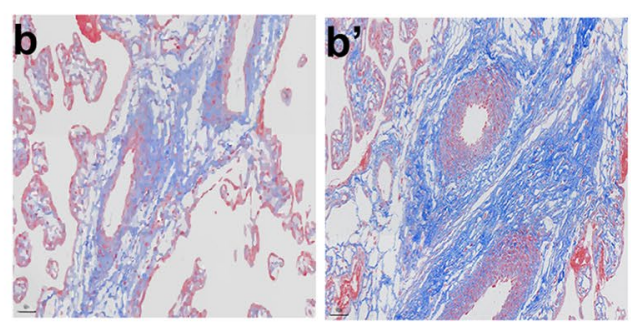

LC3B
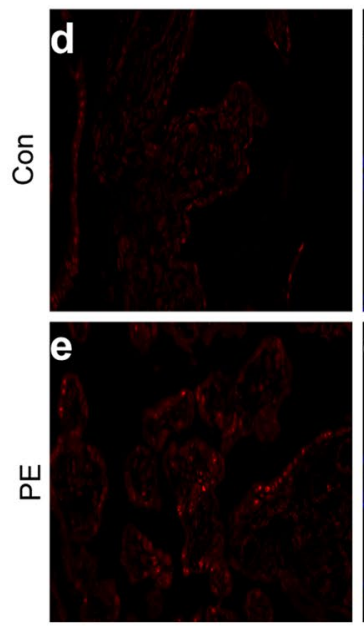

DAPI
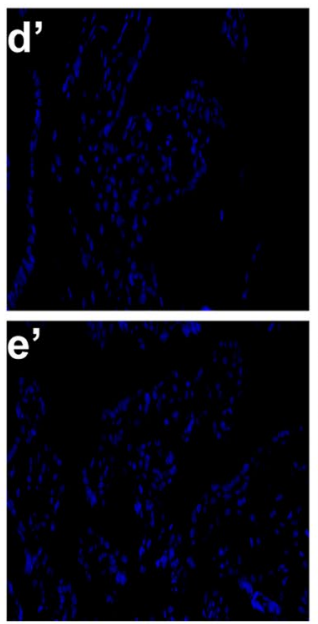

$\mathbf{f}$

Con1 Con2 Con3 PE1 PE2 PE3

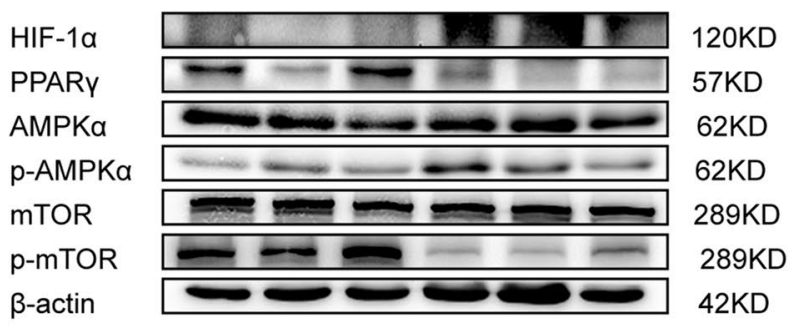

g
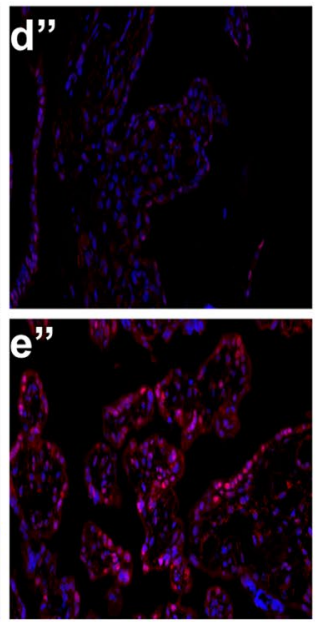

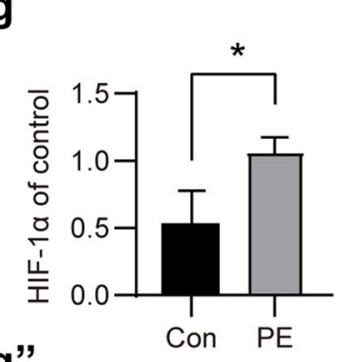

g"

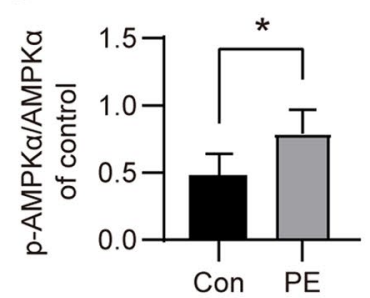

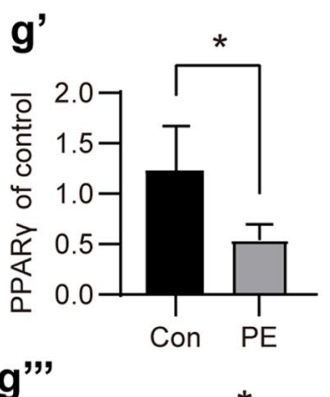

g"'

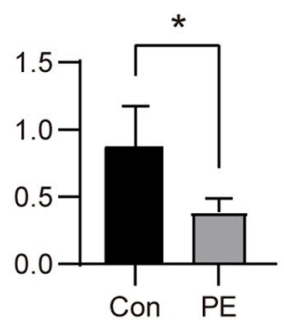

Fig. 1 Increased autophagy in placentas of preeclampsia. (a, $\left.\mathbf{a}^{\prime}\right)$ Histology analysis of H\&E-stained healthy and PE placentas. (b, $\left.\mathbf{b}^{\prime}\right)$ Trichrome Masson staining showing increased fibrosis in PE placenta. (c) The expression levels of LC3B and beta-actin were analyzed using Western blot. $\left(\mathbf{c}^{\prime}\right)$ Plot showing the quantification of relative LC3B expression levels in healthy and PE placentas. Beta-actin expression levels were used for normalization. $\left(\mathbf{d}-\mathbf{e}^{\prime \prime}\right)$ The expressions of

compared to that in the control group (Fig. 1f, $\mathrm{g}^{\prime}, P<0.05$ ), indicating that PE placentas were in a state of hypoxia. Further analysis showed that the phosphorylation level of AMPK $\alpha$ was significantly elevated $(P<0.05)$. On the contrary, the phosphorylation of mTOR was significantly reduced $(P<0.05)$ (Fig. 1f $\left.-\mathrm{g}^{\prime \prime \prime}\right)$. These data suggest that hypoxia induces autophagy by activating AMPK $\alpha$ and inhibiting mTOR in PE human placenta.
LC3B in placentas were validated using immunofluorescence staining. (f) The expression levels of the essential autophagy-related proteins HIF $1 \alpha$, PPAR $\gamma$, AMPK $\alpha, p-A M P K \alpha$, mTOR, and p-mTOR were measured using the Western blot test. $\left(\mathbf{g}-\mathbf{g}^{\prime \prime \prime}\right)$ Plots showing the quantification of HIF1 $\alpha$, PPAR $\gamma$, p-AMPK $\alpha / A M P K \alpha$, and p-mTOR/ mTOR. All data are representative of three independent experiments. $* P<0.05, * * P<0.01$

\section{Esomeprazole inhibits hypoxia-induced autophagy in vitro}

We first tested whether esomeprazole could inhibit autophagy using a cell-based assay approach. Both the transwell migration and wound-healing assay showed that hypoxia and L-NAME $(70 \mu \mathrm{M}, 48 \mathrm{~h})$ treatment inhibited the migration of HTR8/SVneo cells $(P<0.05)$. Esomeprazole treatment strongly rescued the cell migration of HTR8/SVneo cells 


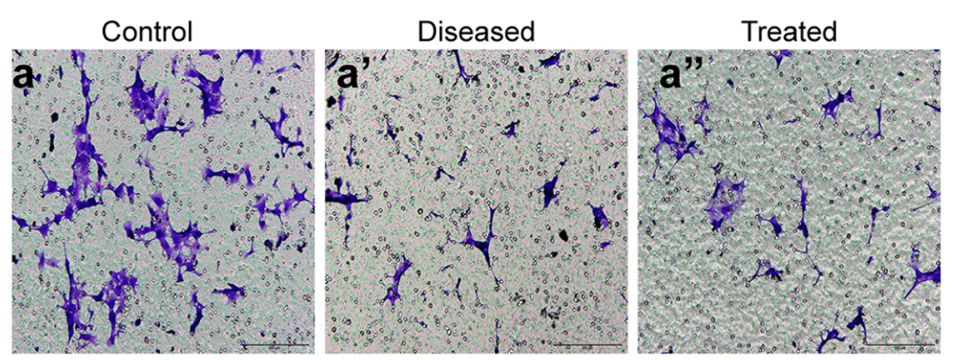

b

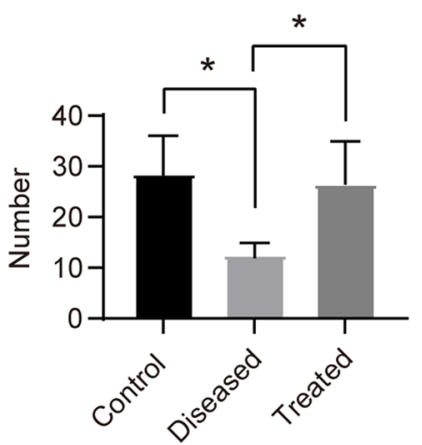

C

Diseased

Treated

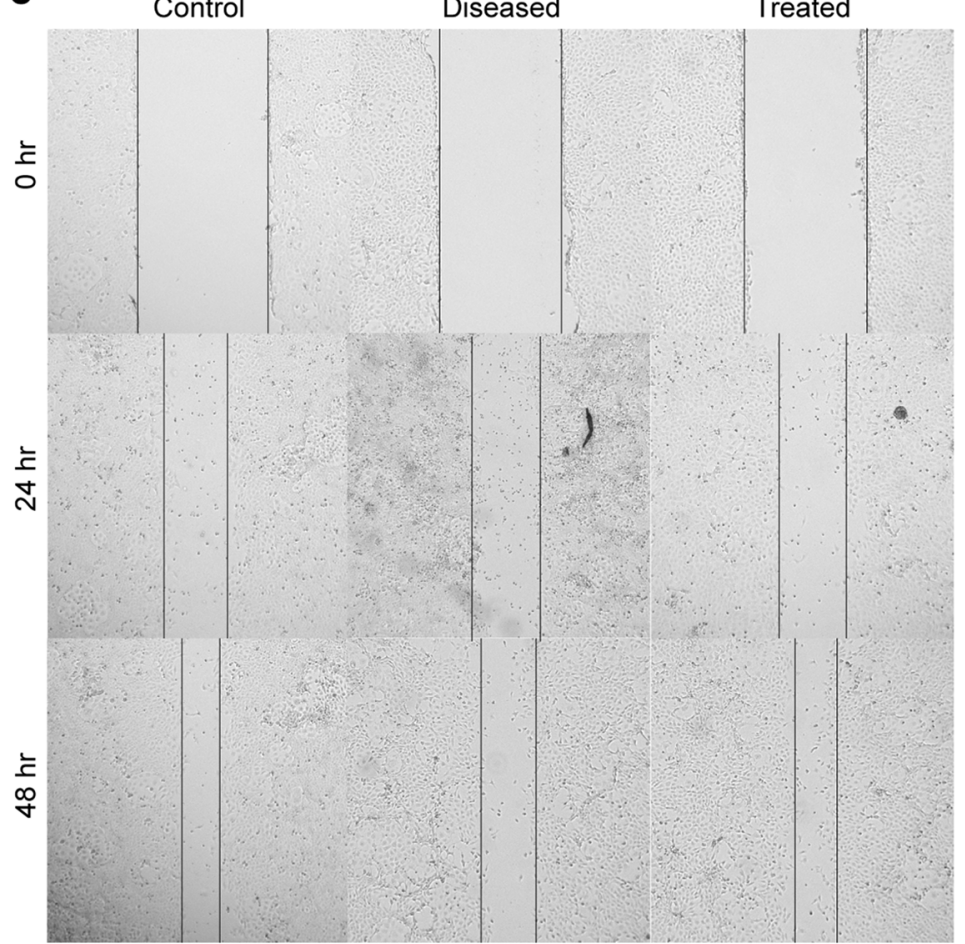

d

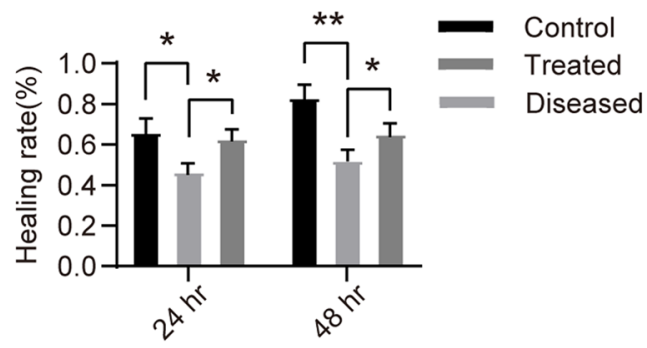

e

\section{Control Diseased Treated}

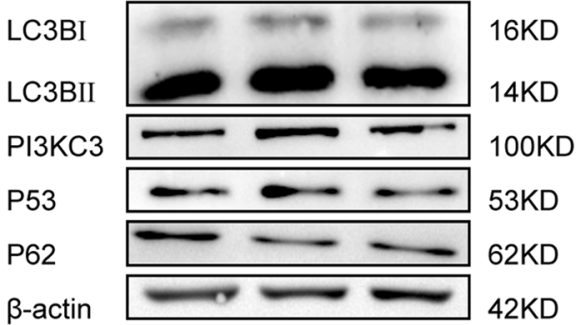

f

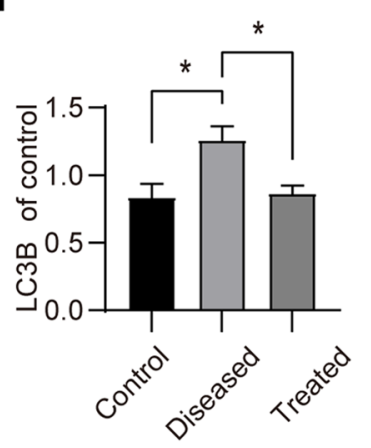

f'

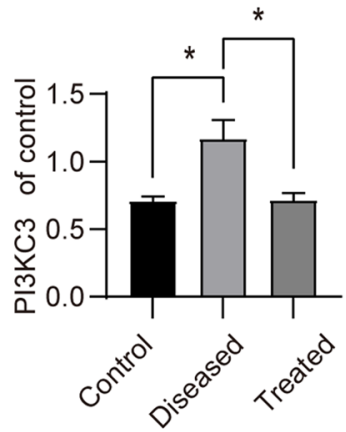

f"

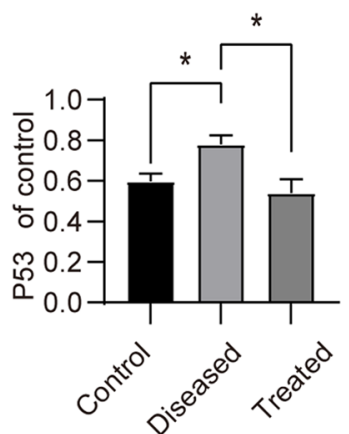

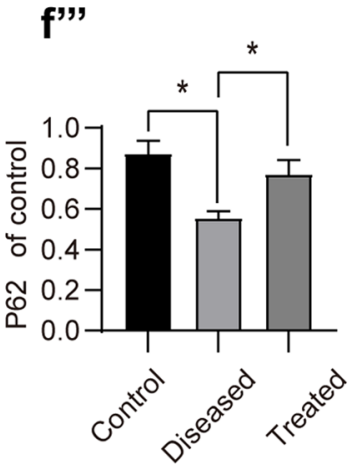

Fig. 2 Esomeprazole suppresses migration and autophagy in HTR-8/ Svneo cells treated with hypoxia and L-NAME. (a, $\left.\mathbf{a}^{\prime}, \mathbf{a}^{\prime \prime}\right)$ HTR-8/ Svneo cells cultured in transwells were first treated with hypoxia and L-NAME, followed by DMSO or esomeprazole treatment. Transmigrated cells were stained with crystal violet and imaged. (b) Plot showing the quantification of the transmigrated cells. (c) HTR-8/ Svneo cells cultured in 6-well plates. After introducing a cell-free

$(P<0.05)$ (Fig. 2a-d). Western blot analysis revealed that LC3B protein levels were elevated in HTR8/SVneo cells gap, the cells were treated with hypoxia and L-NAME, followed by DMSO or esomeprazole treatment. Cell migrations were recorded at $24 \mathrm{~h}$ and $48 \mathrm{~h}$. (d) Plot showing the quantification of the healing rate. (e) LC3B, P53, and PI3KC3 proteins levels were detected via Western blot. (f-f $\left.\mathbf{f}^{\prime \prime \prime}\right)$ Plot depicting the quantification of LC3B, P53, and PI3KC3 expression levels. ${ }^{*} P<0.05, * * P<0.01$

subject to hypoxia and L-NAME treatment $(70 \mu \mathrm{M}, 48 \mathrm{~h})$, compared to cells under normoxia environment $(P<0.05)$. 
Concurrently, the expressions of PI3KC3 and P53 also were increased under hypoxia and L-NAME treatments. Nonetheless, esomeprazole treatment successfully reduced LC3B, P53, and PI3KC3 expression in hypoxia/L-NAME-treated HTR8/SVneo cells (Fig. 2e-f $\mathrm{f}^{\prime \prime \prime}$ ). This was further confirmed by the immunofluorescence staining analysis of LC3B and P53 (Fig. 3a-f"). In addition to HTR8/SVneo cells, we further validated these findings in HUVECs. As expected, HUVECs treated with hypoxia and L-NAME showed impaired cell migration ability, while the esomeprazole treatment effectively restored HUVEC migration (Fig. 4a, b). Moreover, esomeprazole potently suppressed hypoxia and L-NAME treatment-upregulated LC3B and P53 expression (Fig. 4c-i"). Collectively, these data showed that esomeprazole can abrogate hypoxia and L-NAME-induced autophagy.

\section{Esomeprazole reduces autophagy by inhibiting phosphorylation of AMPKa in vitro}

Compared to the phosphorylation of AMPK $\alpha$ under normoxia, the phosphorylation of AMPK $\alpha$ was elevated in HTR8/SVneo cells under hypoxia and L-NAME treatment (70 $\mu \mathrm{M}, 48 \mathrm{~h}$ ) (Fig. 5a-d). On the contrary, the phosphorylation of mTOR was significantly inhibited (Fig. 5d-e'"). Notably, esomeprazole treatment reduced AMPK $\alpha$ phosphorylation and SIRT1 expressions, while the phosphorylation of mTOR was significantly increased (Fig. 5d-e ${ }^{\prime \prime \prime}$ ). We also validated these findings in HUVECs Similar to HTR8/SVneo, HUVECs under hypoxia and L-NAME treatment showed significantly increased phospho-AMPK $\alpha$ and HIF $1 \alpha$ levels and decreased PPAR $\gamma$ and phosphomTOR levels, while they were restored by esomeprazole treatment (Fig. 6a-e $\mathrm{e}^{\prime \prime \prime}$ ). Therefore, these results suggest esomeprazole-inhibited hypoxia and L-NAME treatmentinduced autophagy by activating mTOR and inhibiting AMPK $\alpha$ signaling.

\section{Esomeprazole ameliorates preeclampsia-like symptoms in mice induced by L-NAME treatment}

PE is characterized by significantly increased systolic blood pressure and the onset of proteinuria. The administration of L-NAME successfully led to increased systolic pressure and the presence of protein in the urine (Fig. 7a, b). In addition, L-NAME treatment also increased the sFLT-1 level in serum (Fig. 7c). Esomeprazole treatment significantly reduced systolic pressure, urinary protein, and serum sFLT-1 levels. Furthermore, both H\&E-based histology analysis and Trichrome Masson's staining demonstrated that the esomeprazole treatment effectively preserved placenta structure and reduced intraplacental fibrosis (Fig. 7d-e"). These data strongly indicate that PE symptoms were reversed by esomeprazole treatment.
Esomeprazole protects the placenta from L-NAME-induced autophagy by upregulating PPAR $\gamma$ in vivo

To understand the molecular mechanisms by which esomeprazole inhibits autophagy in L-NAME-treated placenta, we first analyzed the protein level of LC3B. Western blot analysis revealed that $\mathrm{LC} 3 \mathrm{~B}$ protein levels were significantly elevated in the PE group compared to the control group, whereas esomeprazole treatment potently reduced the LC3B levels (Fig. 8a, a'). Comparable results were obtained comparing the LC3B expression level in control, diseased, or treated placentas based on immunofluorescence staining-based assays (Fig. 8b-d"). More importantly, we found that PPAR $\gamma$ protein levels were also decreased in the PE mice placentas $(P<0.05)$. Interestingly, the phosphorylation of AMPK $\alpha$ was elevated in the PE group; however, the phosphorylation of mTOR was significantly inhibited $(P<0.05)$. Esomeprazole inhibited the phosphorylation of AMPK $\alpha$, whereas it increased the phosphorylation of mTOR (Fig. 8e-f $\mathrm{f}^{\prime \prime \prime}$ ).

\section{Discussion}

PE has negative consequences on maternal and fetal health during pregnancy, including increased perinatal mortality, preterm births, infants who are considered small for gestational age, high rates of cesarean deliveries, and other adverse outcomes, even in later postnatal periods (Antza et al. 2018; Gu et al. 2019). The relevant mechanism is not yet fully elucidated. Although many factors impact preeclampsia-including some not yet identified-recent evidence suggests that insufficient trophoblast cell invasion causes uterine spiral artery remodeling disorder and shallow placental implantation (Mol et al. 2016b). This leads to placental ischemia and hypoxia, followed by excessive autophagy.

We found that HIF- $1 \alpha$ was elevated in PE placenta; this only occurs for a long time under hypoxic conditions. In addition to the resulting placental ischemia and hypoxia, bioactive factors released by the mother into the blood circulation cause a systemic inflammatory reaction and vascular endothelial injury, inducing clinical symptoms of PE. Soluble Fms-like tyrosine kinase-1 (sFlt-1), an antagonist of VEGF, has been shown to induce preeclampsia-like disease in rodents (Hastie et al. 2019). This also has been confirmed in our animal experiments. The placenta is undoubtedly involved in the pathogenesis, considering that termination of the pregnancy can eliminate the disease.

In vitro, our findings indicate that autophagy was increased in PE placenta. During the early pregnancy period, a low-oxygen environment in the placenta is beneficial for the differentiation of trophoblast cells from trophoblastic stem cells, promoting trophoblastic cell 
Fig. 3 Esomeprazole suppresses autophagy in HTR-8/Svneo cells treated with hypoxia and L-NAME. (a-f') The expressions of LC3B and P53 were measured by immunofluorescence staining. All data are representative of three independent experiments

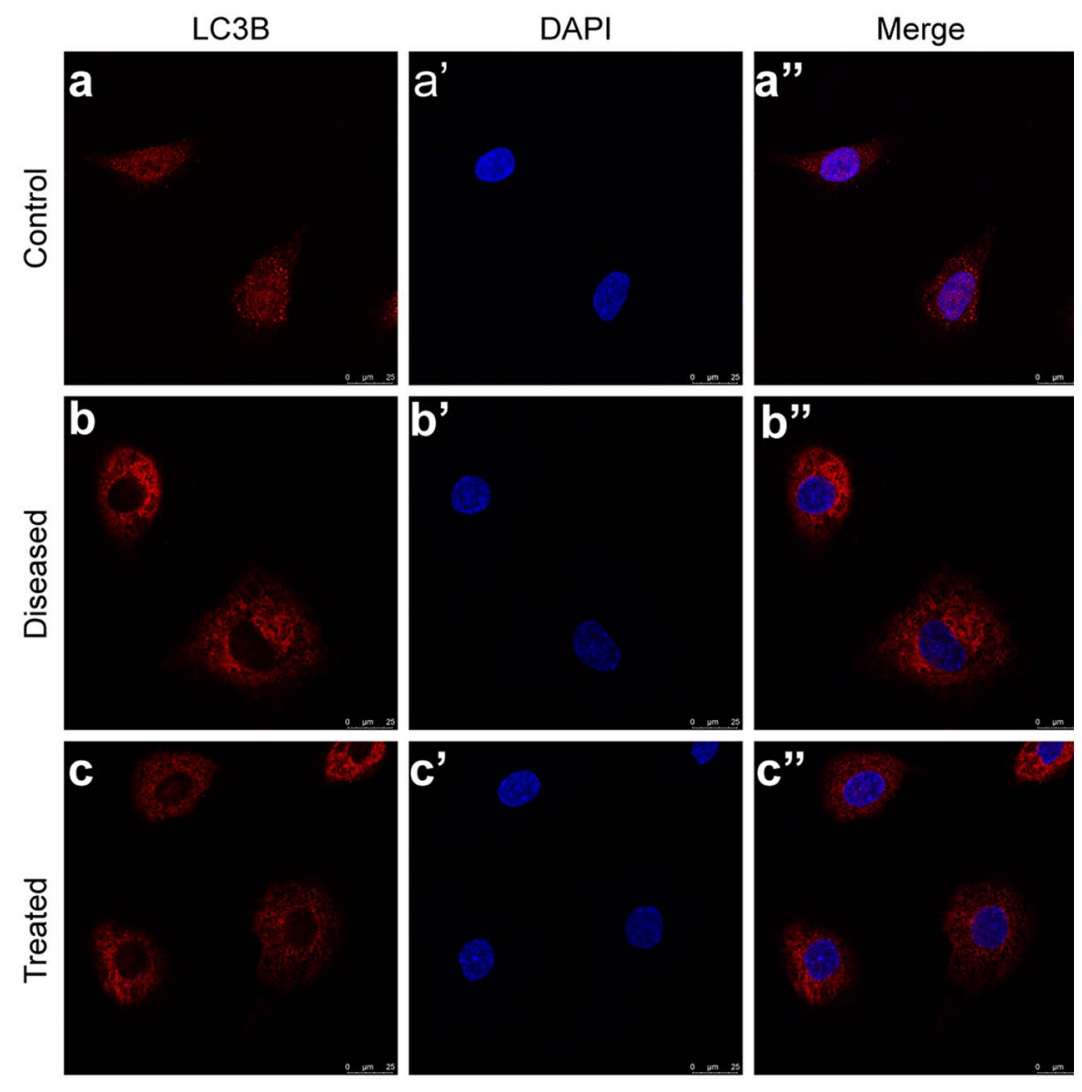

P53

DAPI
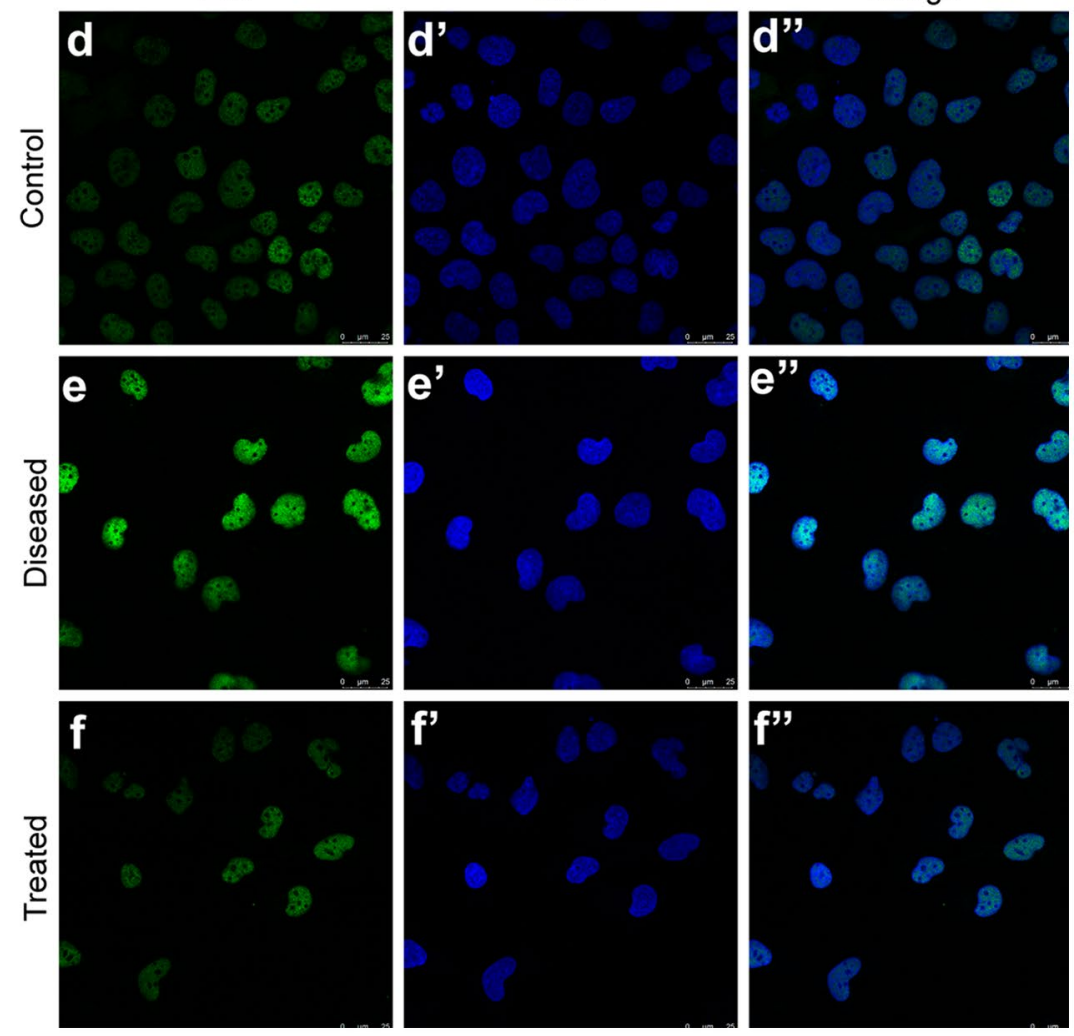

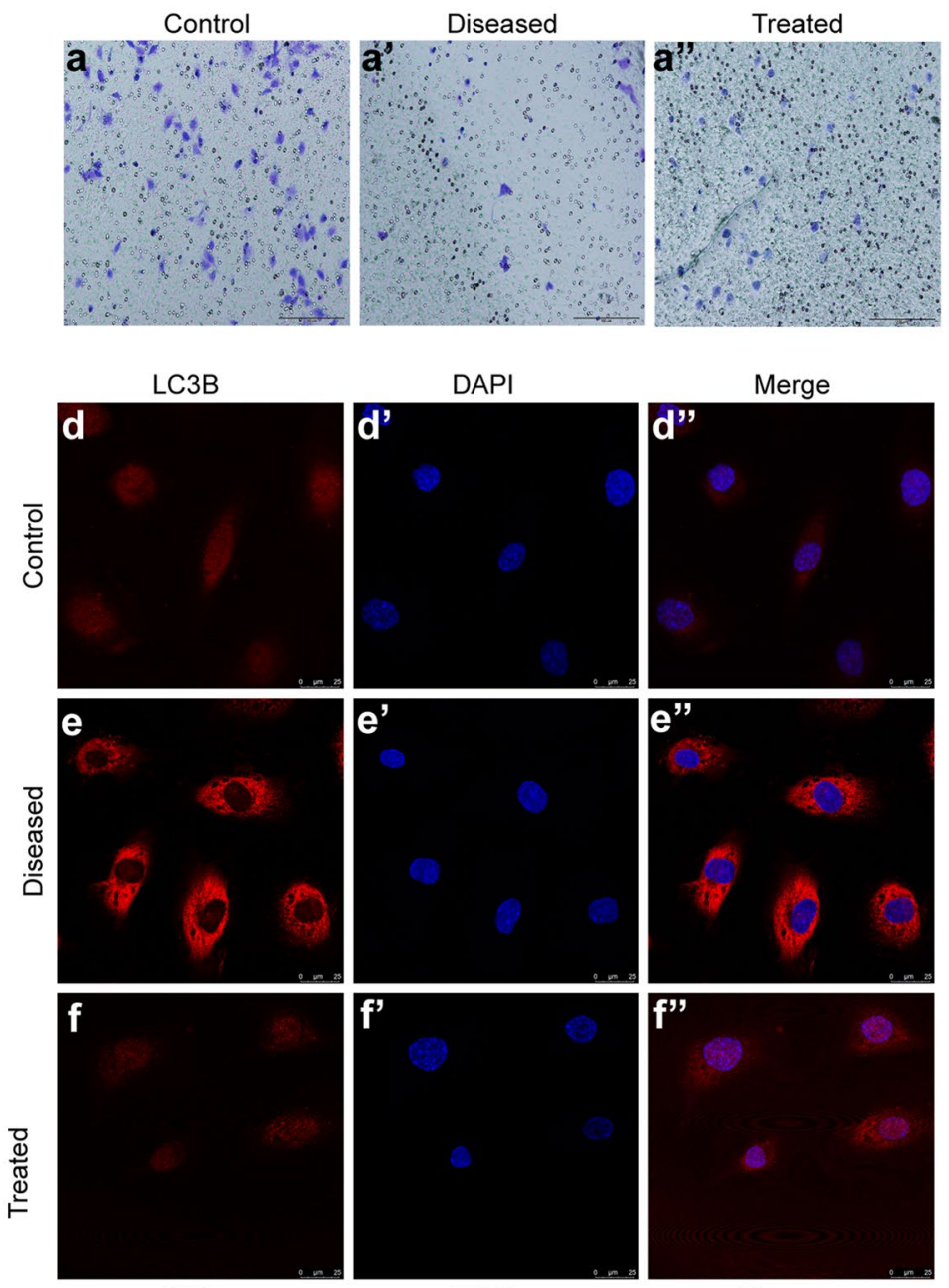

P53

DAPI
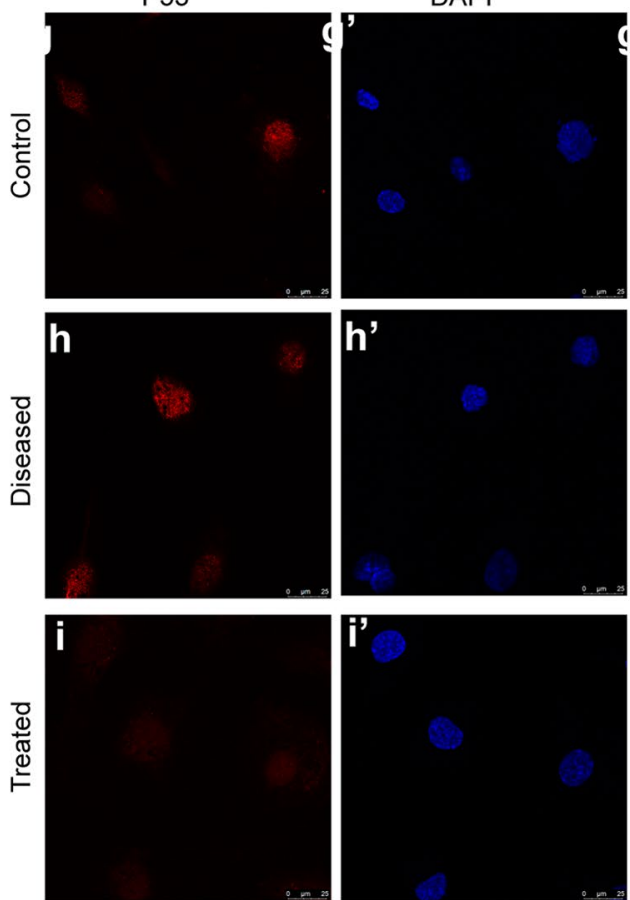

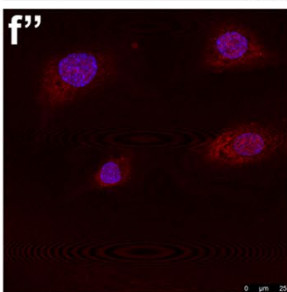

Merge
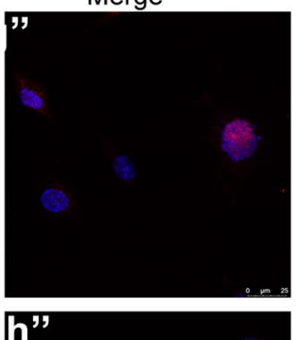

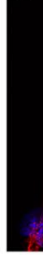

(4)
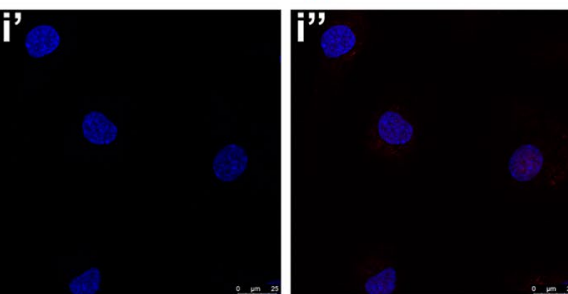

LC3BI

LC3BII

$\beta$-actin

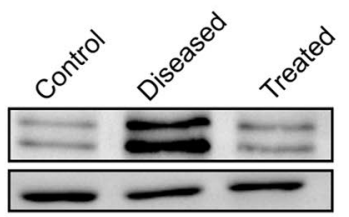

$14 \mathrm{KD}$

$16 K D$

$42 K D$

$c^{\prime}$

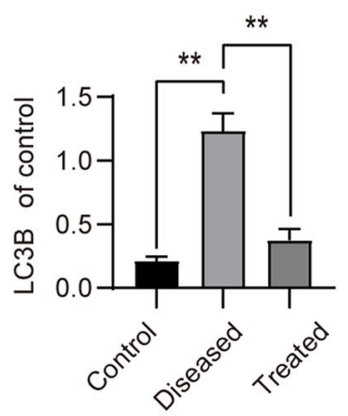


4Fig. 4 Esomeprazole suppresses autophagy in HUVEC treated with hypoxia and L-NAME. (a, a', a") Cell migrations upon hypoxia and L-NAME treatment were measured using transwell-migration assays. (b) Quantification of cell migration of (a, $\left.\mathbf{a}^{\prime}, \mathbf{a}^{\prime \prime}\right)$. (c) LC3B proteins levels were detected via Western blot. $\left(\mathbf{c}^{\prime}\right)$ Plot depicting the quantification of LC3B expression levels. (d-i") The LC3B and P53 expressions were visualized using immunofluorescence staining. All data are representative of three independent experiments: $* P<0.05$, $* * P<0.01$

migration and remolding spiral arteries during this period in humans (Nakashima et al. 2017). Under these conditions, autophagy is activated (Yamanaka-Tatematsu et al. 2013). Proper autophagy is necessary to regulate protein quality control and maintain intracellular homeostasis (Levine and Kroemer 2019). However, excessive autophagy induced by long-term anoxia can lead to cell death and affect placental function. To better simulate the pathological conditions of preeclampsia, cells were treated by hypoxia and L-NAME treatment for $48 \mathrm{~h}$, followed by the rapid activation of autophagy. Excessive autophagy disrupts invasion and vascular remodeling under hypoxia. We found that, with increased HIF-1 $\alpha$ expression, placental autophagy
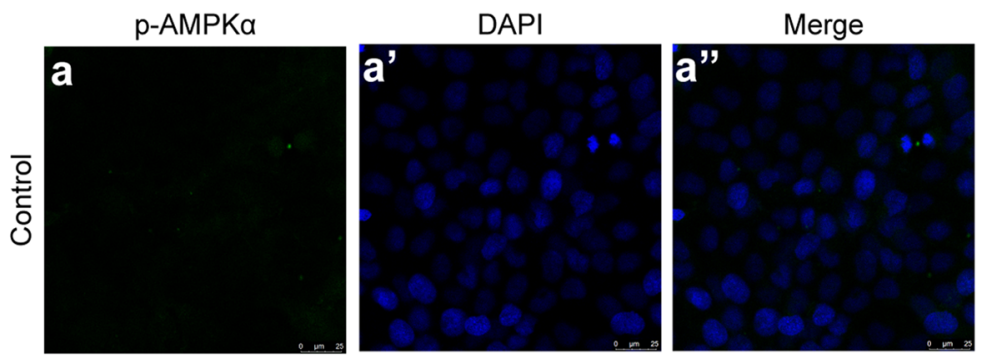

d
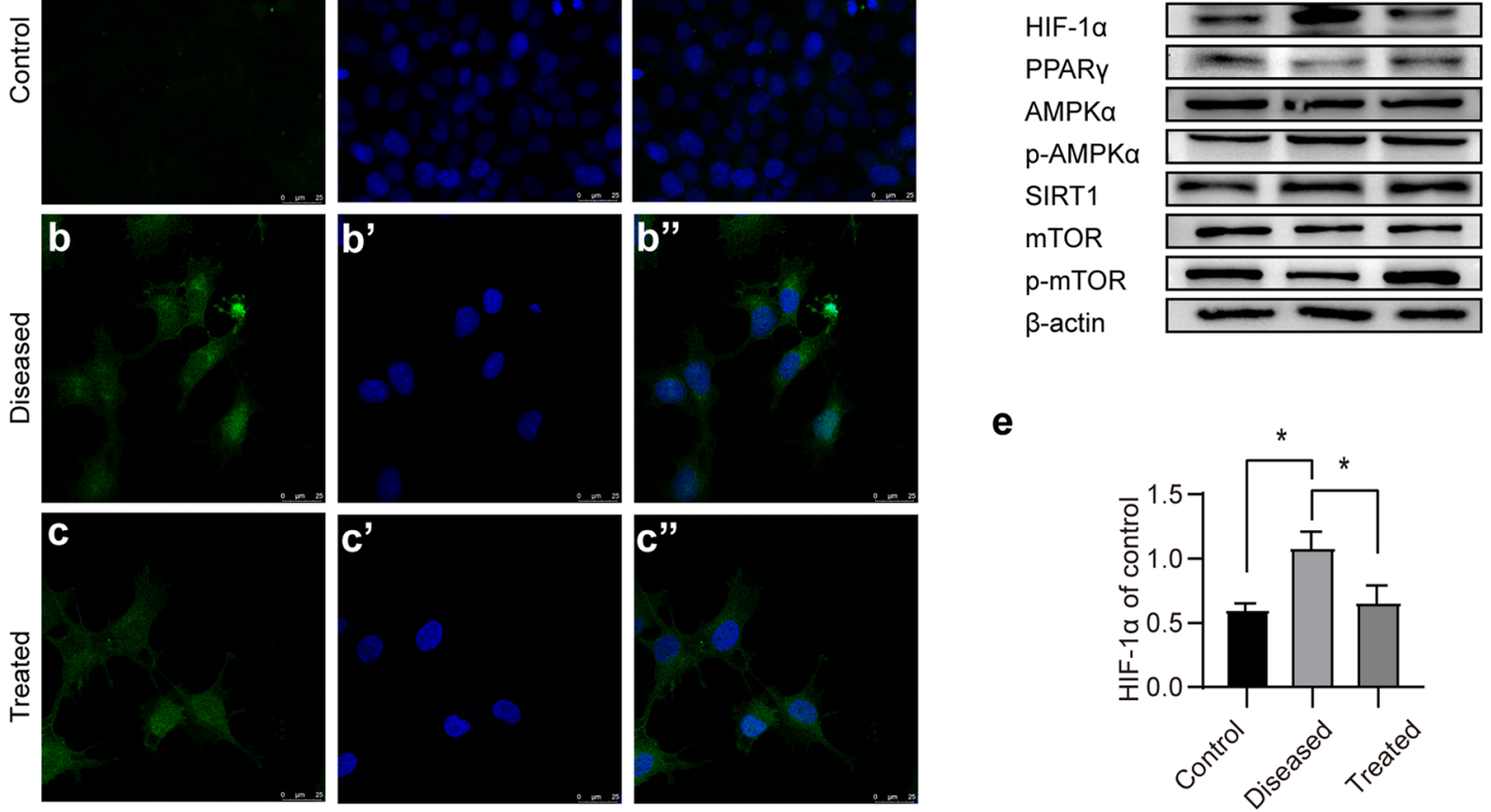

$120 K D$

$57 \mathrm{KD}$

$62 K D$

$62 \mathrm{KD}$

120KD

289KD

$289 K D$

$\beta$-actin

$42 K D$

e

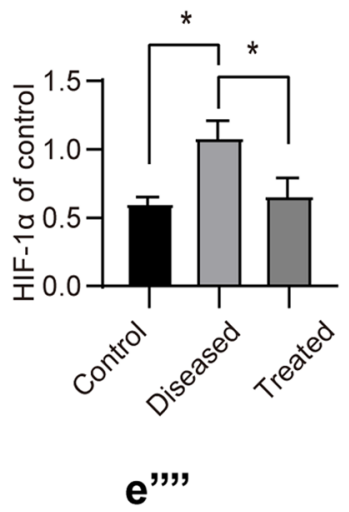

$\mathbf{e}^{\prime}$

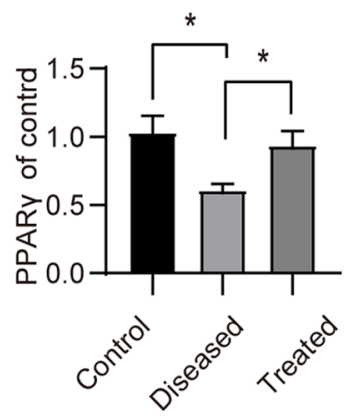

$e^{\prime \prime}$

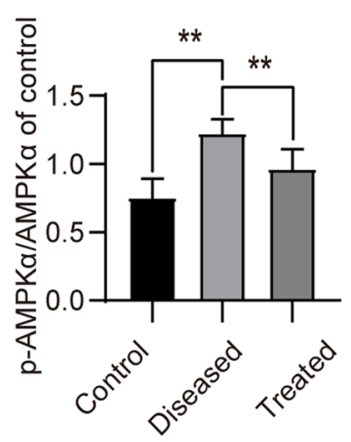

Fig. 5 Esomeprazole inhibits autophagy in HTR-8/Svneo cells by suppressing the SIRT1/AMPK $\alpha$ pathway. (a-c") Immunofluorescence staining of $\mathrm{p}$-AMPK $\alpha$ in control or esomeprazole-treated HTR-8/Svneo. (d) Western blot analysis of the expression levels of $\mathrm{p}-\mathrm{AMPK} \alpha, \mathrm{AMPK} \alpha, \mathrm{PPAR} \gamma, \mathrm{SIRT} 1, \mathrm{mTOR}$, and $\mathrm{p}-\mathrm{mTOR}$ in con-

$e^{\prime \prime \prime}$
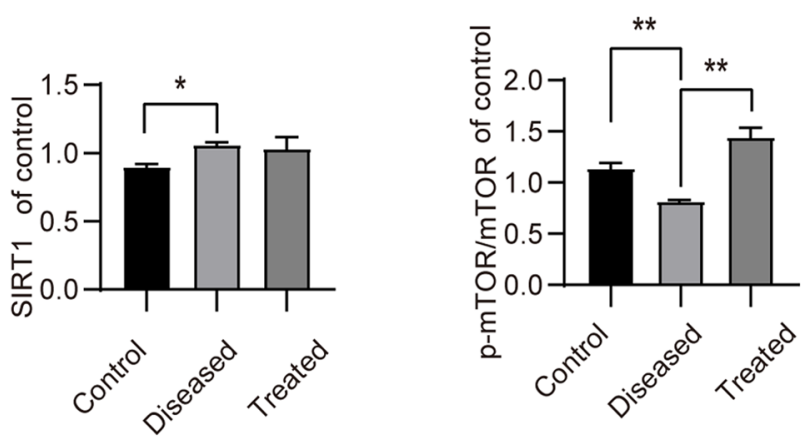

trol or esomeprazole-treated HTR-8/Svneo. $\left(\mathbf{e}-\mathbf{e}^{\prime \prime \prime \prime \prime}\right)$ Plots showing the quantification of HIF $1 \alpha, \operatorname{PPAR} \gamma, \mathrm{p}-\mathrm{AMPK} \alpha / \mathrm{AMPK} \alpha$, and p-mTOR/ mTOR. All data are representative of three independent experiments: $* P<0.05, * * P<0.01$ 

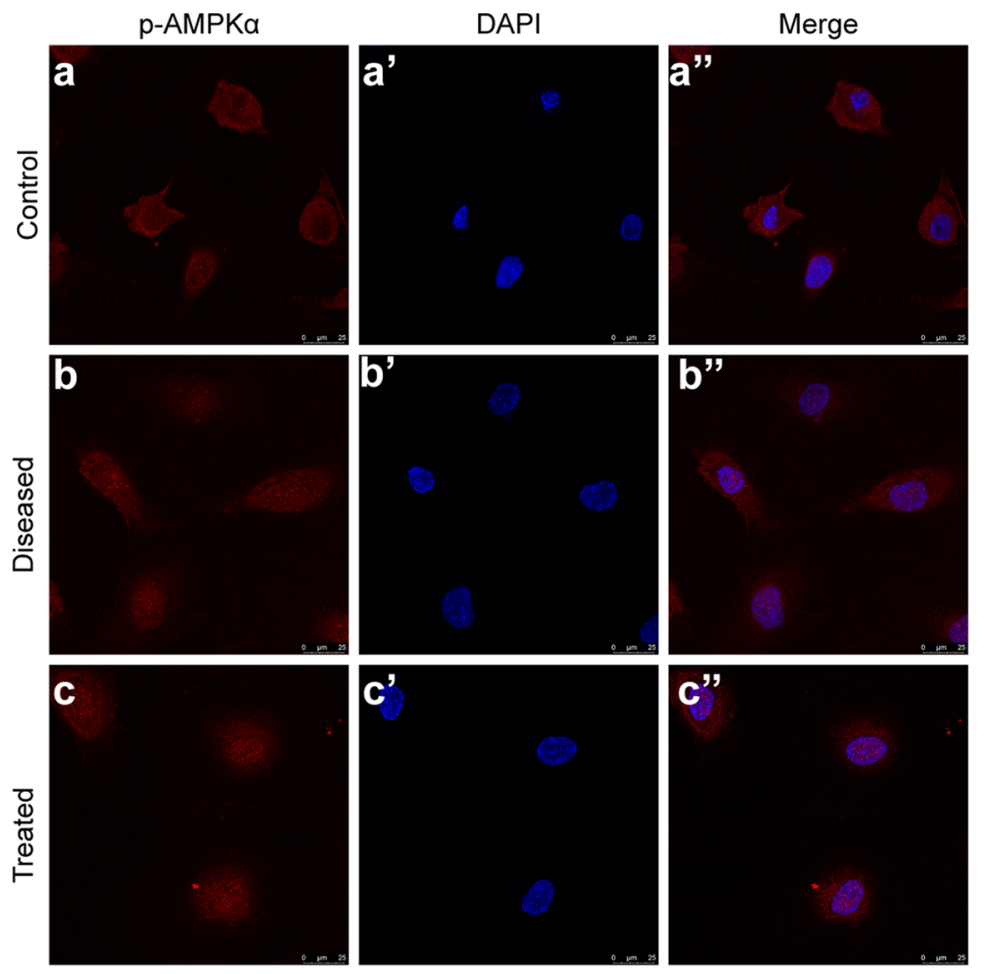

d
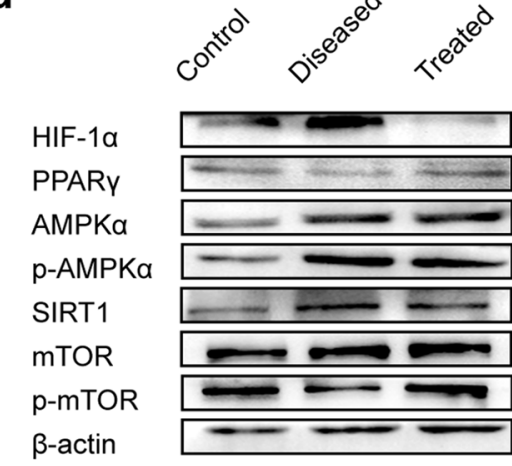

120KD

$57 \mathrm{KD}$

$62 K D$

$62 \mathrm{KD}$

$120 K D$

$289 K D$

$289 K D$

$42 K D$

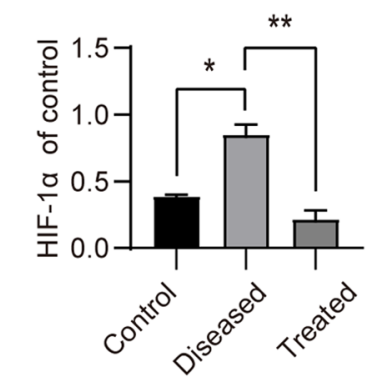

e
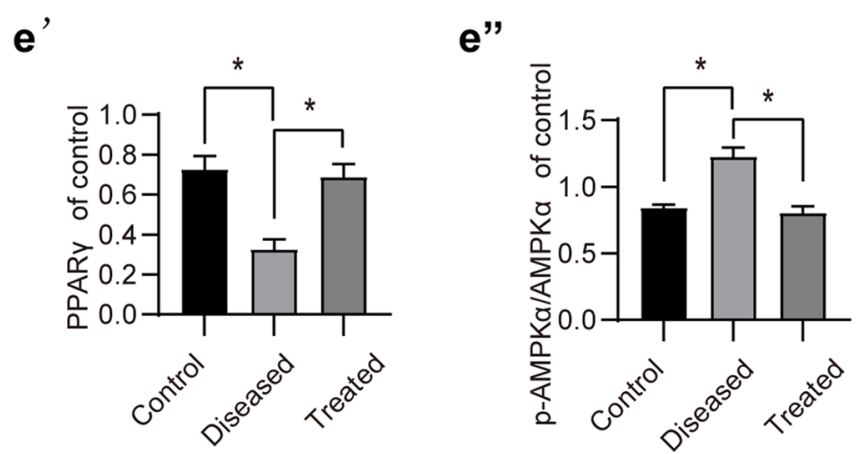

$e^{g 9}$

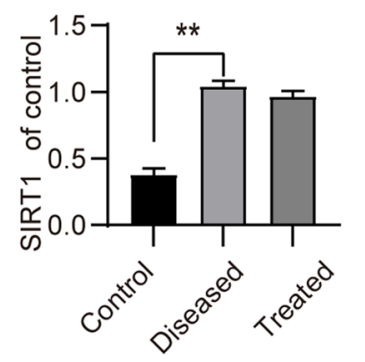

$e^{\text {"M" }}$

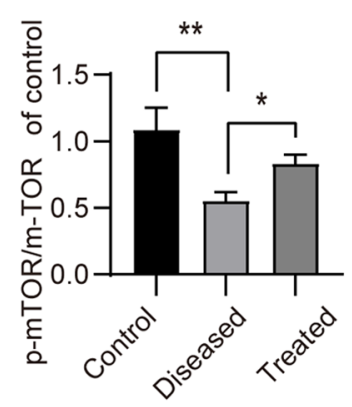

Fig. 6 Esomeprazole inhibits autophagy via SIRT1/AMPK $\alpha$ pathway in HUVECs. $\left(\mathbf{a}-\mathbf{c}^{\prime \prime}\right)$ Expression levels of p-AMPK $\alpha$ in HUVECs were visulized by immunofluorescence staining. (d) The protein levels of HIF1 $\alpha$, phospho-AMPK $\alpha$, AMPK $\alpha$, PPAR $\gamma$, SIRT1, mTOR, and
phospho-mTOR were measured using Western blotting. (e-e $\left.\mathbf{e}^{\prime \prime \prime \prime}\right)$ Plot showing the quantification of HIF1 $\alpha$, PPAR $\gamma$, SIRT1, and phosphomTOR protein levels in HUVECs. All data are representative of three independent experiments: $* P<0.05, * * P<0.01$

kinase that regulates energy metabolism (Kim and Lee 2014). It is expressed in various metabolism-related organs and can be activated via various stimuli, including cellular stress, exercise, and numerous hormones and substances that affect cellular metabolism. AMPK activation is dependent on the deacetylation of the lysine of liver kinase B1 residue by SIRT1 (Ganesan et al. 2017). In our study, SIRT1 also was found to be highly activated in the PE placenta. SIRT1 exerts its cellular autonomic function by regulating many transcription factors, such as p53, in the nucleus. SIRT1 controls cellular-repair mechanisms, such as autophagy and mitochondrial biogenesis. The phosphorylation of AMPK $\alpha$ governs the formation of autophagy vesicles by the PI3KC3 
Fig. 7 Esomeprazole ameliorates the preeclampsia symptoms caused by L-NAME. (a) Plot showing the SBP of control, L-NAME-treated, or L-NAME + esomeprazoletreated mice were measured. (b) Plot showing the urinary protein concentrations of the control, L-NAME-treated, or L-NAME + esomeprazole-treated mice were determined. (c) The sFLT-1 concentrations in the serum of control, L-NAME, or L-NAME + esomeprazole-treated mice were determined at GD18.5 using ELISA. (d, d', d") Histological analysis of the placentas of control, L-NAME-treated, or L-NAME + esomeprazole-treated mice using $\mathrm{H} \& \mathrm{E}$ staining. (e, $\left.\mathbf{e}^{\prime}, \mathbf{e}^{\prime \prime}\right)$ Fibrosis in the placentas of control, L-NAME-treated, or L-NAME+ esomeprazole-treated mice were measured using Trichrome Masson staining. A blue color in the square indicates collagen deposition, $N=3$ mice per group, $* P<0.05, * * P<0.01$

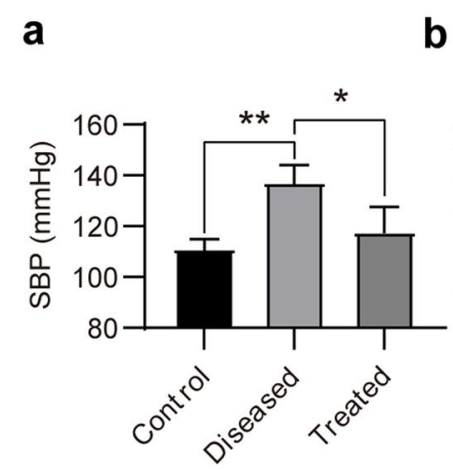

b
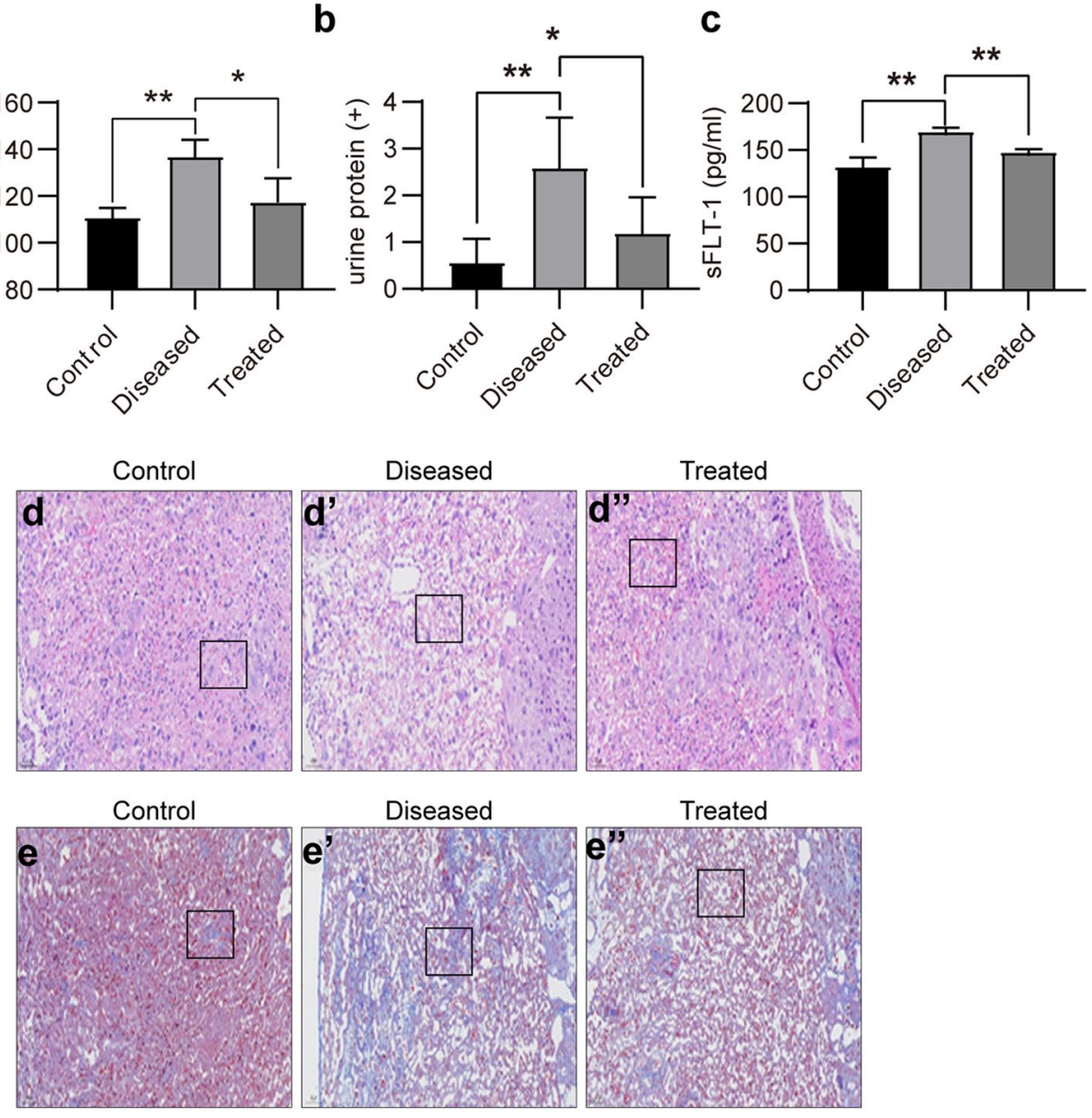

complex (Hurley and Young 2017). Additionally, we found that the SIRT1-dependent activation of AMPK downregulates mTOR, which then initiates a cellular stress response that includes autophagy (Xu et al. 2012). The phosphorylation of mTOR increases the formation of the MTORC1 and mTORC2 complexes. The inhibition of mTORC1 activates autophagy, whereas its activation reduces autophagy. Our study provides evidence that, in the placenta, AMPK and mTOR are engaged in regulating autophagy. We found that excessive autophagy decreased the invasion of HTR8/SVneo. In addition, the hypoxia-induced phosphorylation of AMPK $\alpha$ reduces the expression of PPAR $\gamma$. PPAR $\gamma$ is regulated in part by HIF- $1 \alpha$ (Tache et al. 2013). PPAR- $\gamma$ is an important regulator of spiral artery development and placental function (Liu et al. 2017). Its participation has been noted in the pathogenesis of intrauterine growth retardation and preeclampsia. Evidence suggests that PE placental autophagy is regulated by hypoxia-induced AMPK $\alpha$ phosphorylation.

Esomeprazole, a proton-pump inhibitor, is a drug widely used to treat gastroesophageal reflux disease, a common condition associated with pregnancy (Galmiche et al. 2011). It has been shown that patients receiving esomeprazole experience less gestational hypertension and lower plasma ENG and sFLT-1 levels (Onda et al. 2017). Our animal experiments confirm this. A prospective Dutch cohort study treated 430 pregnant women with preeclampsia with PPIs, alpha methyldopa, steroid hormones, ferrous fumarate, polyethylene glycol, and nifedipine. This study found that PPIs reduced the level of sFlt-1 level and sENG and prolonged the week of pregnancy effectively (Saleh et al. 2017). Previous research also found that PPIs could upregulate the key placental protective enzyme, heme-oxygenase 1 , and then improved the maternal antioxidant-defense function. Furthermore, esomeprazole also mitigates tumor necrosis factor- $\alpha$-induced endothelial dysfunction. Esomeprazole reduces expression of endothelial vascular cell adhesion molecule 1, prevents leukocyte adherence to endothelium, and promotes angiogenesis (Brownfoot et al. 2016; Kaitu'uLino et al. 2017). In our PE mice model, we also found that esomeprazole decreased sFlt-1 serum levels. It has been reported that heme-oxygenase 1 (HO-1) may decrease sFlt-1 secretion (Cudmore et al. 2007), although Tong et al. came to the opposite conclusion (Tong et al. 2015). Moreover, researchers found combining esomeprazole with other drugs, 
a
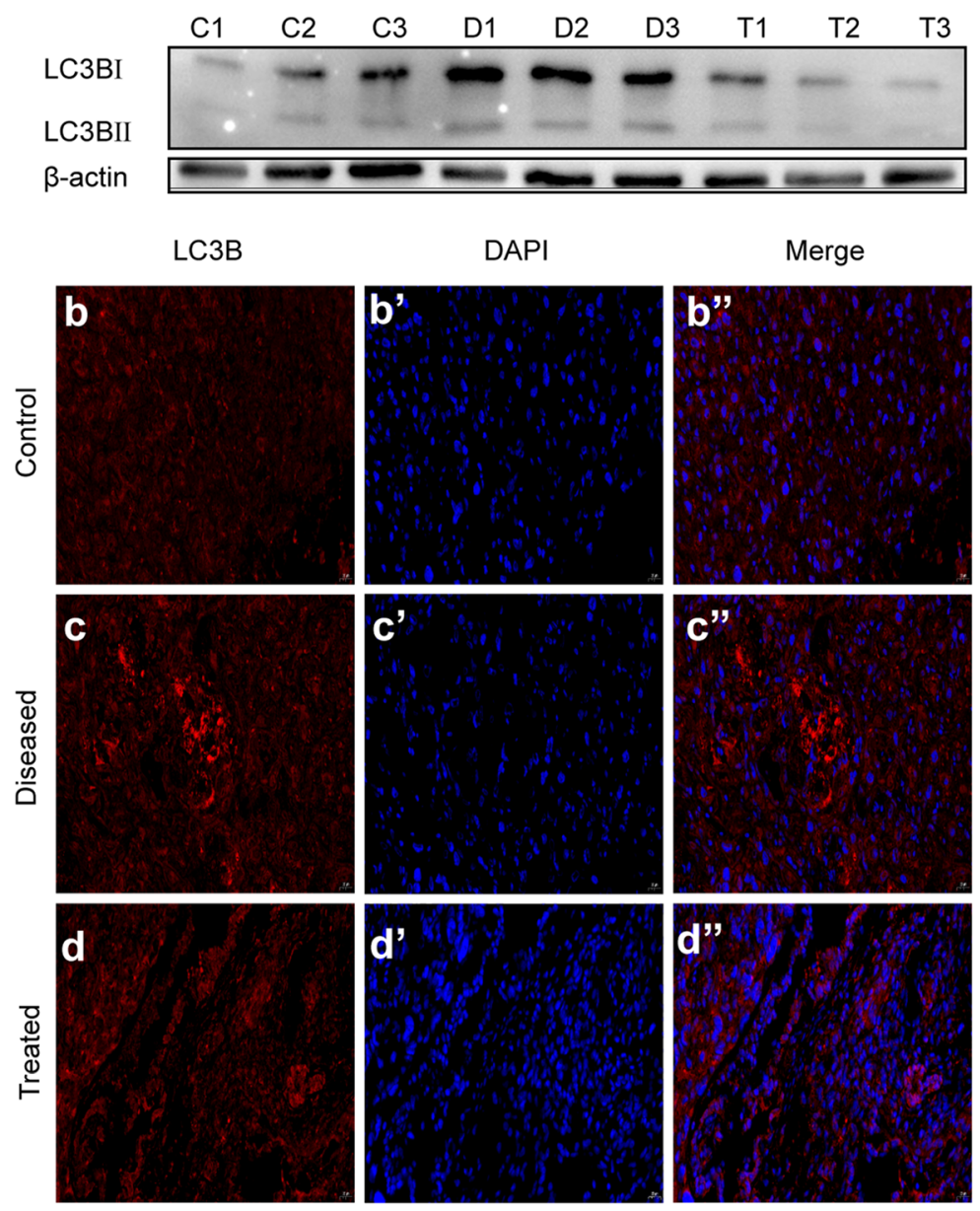

e
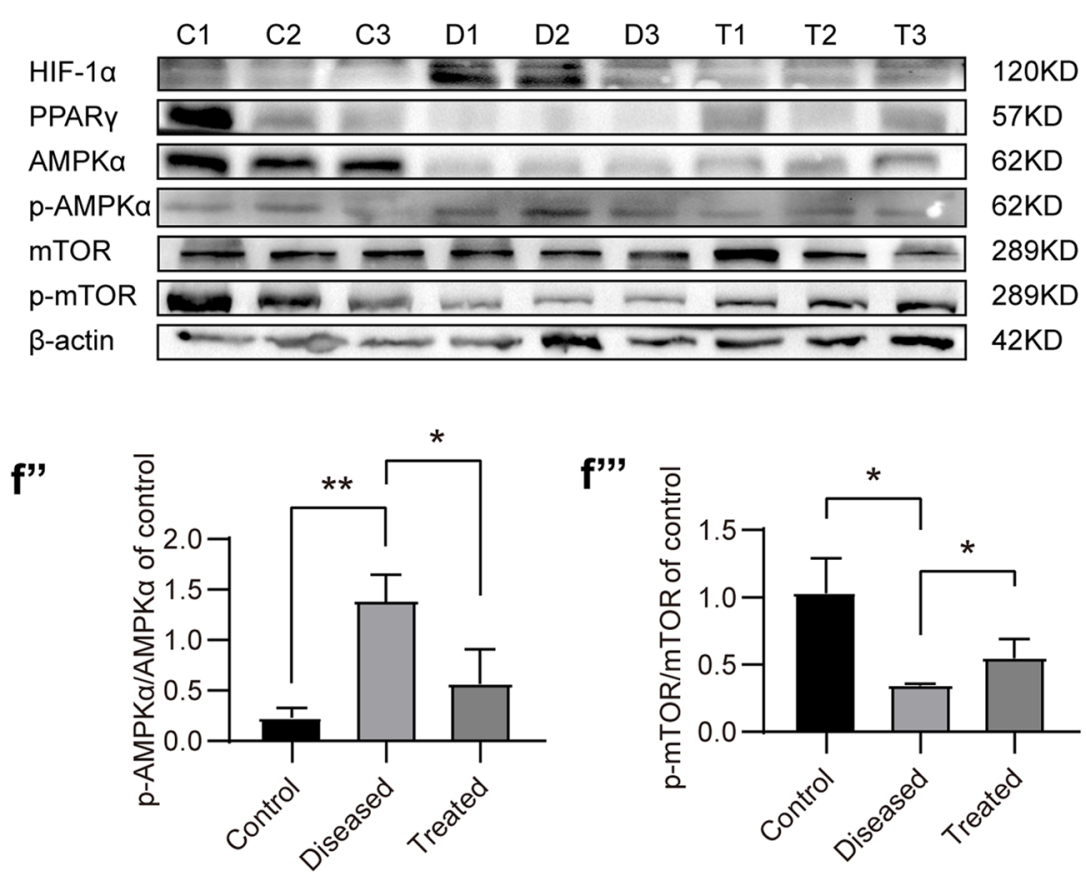

$\mathbf{a}^{\prime}$

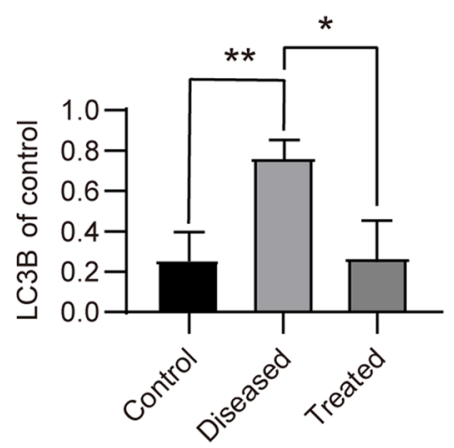

f

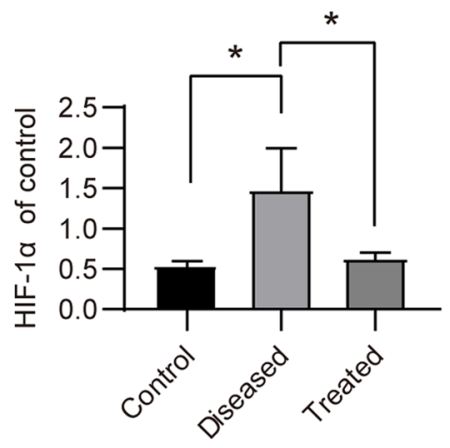

f

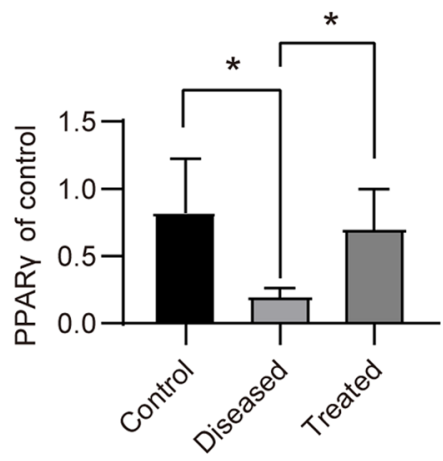


४Fig. 8 Esomeprazole-suppressed autophagy induced by L-NAME via $\mathrm{AMPK} \alpha-\mathrm{mTOR}$ in PE mice. (a) LC3B proteins levels in placentas of control, L-NAME-treated, or L-NAME + esomeprazoletreated mice were detected by Western blot. $\left(\mathbf{a}^{\prime}\right)$ Plot depicting the quantification of LC3B protein levels. (b-d") LC3B in placentas of control, L-NAME-treated, or L-NAME + esomeprazole-treated mice were evaluated by immunofluorescence staining. (e) The protein levels of phospho-AMPK $\alpha$, AMPK $\alpha$, PPAR $\gamma$, SirT1, mTOR, and phospho-mTOR in placentas of control, L-NAME-treated, or L-NAME + esomeprazole-treated mice were detected by Western blot. (f- $\left.\mathbf{f}^{\prime \prime \prime \prime}\right)$ Quantification of the LC3B, HIF1 $\alpha$, pAMPK $\alpha /$ AMPK $\alpha$, PPAR $\gamma$, and phospho-mTOR/mTOR in placentas of control, L-NAME-treated, or L-NAME + esomeprazole-treated mice, $N=3$ mice per group, $* P<0.05, * * P<0.01$

such as metformin and sulfasalazine, in lower concentrations caused an additive reduction in sFlt- 1 secretion in primary cytotrophoblasts, placental explants, and endothelial cells (Binder et al. 2020; Kaitu'u-Lino et al. 2018). Additionally, with treatment of esomeprazole, in the aortic tissues of pregnant L-NAME-treated mice, autophagy was inhibited (Zhang et al. 2021). This result is similar to ours, finding that placental autophagy was inhibited with the treatment of esomeprazole. Therefore, we hypothesize that esomeprazole treatment may reduce the autophagy of trophoblasts and endothelial cells, thereby preventing the release of sFLT-1 into maternal blood. In vitro, the autophagy of HTR-8/SVneo cells and HUVEC cells induced by hypoxia and L-NAME treatment was inhibited by the esomeprazole treatment. Under the esomeprazole treatment, the expression of HIF- $1 \alpha$ and the activation of AMPK were reduced in vivo and in vitro. This constitutes evidence that cell hypoxia and metabolic abnormalities were relieved. Esomeprazole may prevent autophagy induced by hypoxia.

\section{Conclusion}

The PE placenta experiences excessive autophagy as a result of long-term anoxic conditions. This autophagy contributes to PE symptoms. Autophagy is regulated by the SIRT1/ AMPK $\alpha$-mTOR pathway, upon which esomeprazole acts. Thus, esomeprazole can suppress preeclampsia-like symptoms by inhibiting excessive placental autophagy in PE, acting via the SIRT1/AMPK $\alpha$-mTOR pathway. Furthermore, esomeprazole may increase the expression of PPAR $\gamma$, thereby protecting placental function.

Acknowledgements The work was supported by the staff of the imaging core facility.

Funding The work was supported by the National Natural Science Foundation of China (81873816 and 82071629), Shanghai Development Center (SHDC2020CR6021), Shanghai Municipal Health Commission (202040128), and the Natural Science Foundation of Pudong Municipal Health Commission of Shanghai (PW2019D-13).

\section{Declarations}

Ethical approval This study was performed in line with the principles of the Declaration of Helsinki. Approval was granted by the Ethics Committee of Shanghai First Maternity and Infant Hospital (Approval No. KS1957).

Informed consent Written informed consent was obtained from each patient.

Conflict of interest The authors declare no competing interests.

Open Access This article is licensed under a Creative Commons Attribution 4.0 International License, which permits use, sharing, adaptation, distribution and reproduction in any medium or format, as long as you give appropriate credit to the original author(s) and the source, provide a link to the Creative Commons licence, and indicate if changes were made. The images or other third party material in this article are included in the article's Creative Commons licence, unless indicated otherwise in a credit line to the material. If material is not included in the article's Creative Commons licence and your intended use is not permitted by statutory regulation or exceeds the permitted use, you will need to obtain permission directly from the copyright holder. To view a copy of this licence, visit http://creativecommons.org/licenses/by/4.0/.

\section{References}

Antza C, Cifkova R, Kotsis V (2018) Hypertensive complications of pregnancy: a clinical overview. Metabolism 86:102-111

Bellot G, Garcia-Medina R, Gounon P, Chiche J, Roux D, Pouysségur J, Mazure NM (2009) Hypoxia-induced autophagy is mediated through hypoxia-inducible factor induction of BNIP3 and BNIP3L via their BH3 domains. Mol Cell Biol 29:2570-2581

Binder NK, Brownfoot FC, Beard S, Cannon P, Nguyen TV, Tong S, Kaitu'u-Lino TJ, Hannan NJ (2020) Esomeprazole and sulfasalazine in combination additively reduce sFlt-1 secretion and diminish endothelial dysfunction: potential for a combination treatment for preeclampsia. Pregnancy Hypertens 22:86-92

Boya P, Codogno P, Rodriguez-Muela N (2018) Autophagy in stem cells: repair, remodelling and metabolic reprogramming. Development 145

Brownfoot FC, Tong S, Hannan NJ, Hastie R, Cannon P, Kaitu'u-Lino TJ (2016) Effects of simvastatin, rosuvastatin and pravastatin on soluble fms-like tyrosine kinase 1 (sFlt-1) and soluble endoglin (sENG) secretion from human umbilical vein endothelial cells, primary trophoblast cells and placenta. BMC Pregnancy Childbirth 16:117

Cluver CA, Hannan NJ, van Papendorp E, Hiscock R, Beard S, Mol BW, Theron GB, Hall DR, Decloedt EH, Stander M, Adams KT, Rensburg M, Schubert P, Walker SP, Tong S (2018) Esomeprazole to treat women with preterm preeclampsia: a randomized placebo controlled trial. Am J Obstet Gynecol 219:388.e1-88.e17

Cudmore M, Ahmad S, Al-Ani B, Fujisawa T, Coxall H, Chudasama K, Devey LR, Wigmore SJ, Abbas A, Hewett PW, Ahmed A (2007) Negative regulation of soluble Flt-1 and soluble endoglin release by heme oxygenase-1. Circulation 115:1789-1797

Galmiche JP, Hatlebakk J, Attwood S, Ell C, Fiocca R, Eklund S, Långström G, Lind T, Lundell L (2011) Laparoscopic antireflux surgery vs esomeprazole treatment for chronic GERD: the LOTUS randomized clinical trial. JAMA 305:1969-1977

Ganesan R, Hos NJ, Gutierrez S, Fischer J, Stepek JM, Daglidu E, Krönke M, Robinson N (2017) Salmonella Typhimurium disrupts 
Sirt1/AMPK checkpoint control of mTOR to impair autophagy. PLoS Pathog 13:e1006227

Glick D, Barth S, Macleod KF (2010) Autophagy: cellular and molecular mechanisms. J Pathol 221:3-12

Gu S, Shen H, Zhou Y, Ni J, Zheng T, Mou Z, Hua X (2019) Tetramethylpyrazine reduces the consequences of nitric oxide inhibition in pregnant rats. J Cell Physiol 234:19799-19806

Hastie R, Brownfoot FC, Pritchard N, Hannan NJ, Cannon P, Nguyen V, Palmer K, Beard S, Tong S, Kaitu'u-Lino TJ (2019) EGFR (Epidermal growth factor receptor) signaling and the mitochondria regulate sFlt-1 (soluble FMS-like tyrosine kinase-1) Secretion. Hypertension 73:659-670

Hiby SE, Apps R, Sharkey AM, Farrell LE, Gardner L, Mulder A, Claas FH, Walker JJ, Redman CW, Morgan L, Tower C, Regan L, Moore GE, Carrington M, Moffett A (2010) Maternal activating KIRs protect against human reproductive failure mediated by fetal HLA-C2. J Clin Invest 120:4102-4110

Hurley JH, Young LN (2017) Mechanisms of autophagy initiation. Annu Rev Biochem 86:225-244

James JL, Stone PR, Chamley LW (2005) Cytotrophoblast differentiation in the first trimester of pregnancy: evidence for separate progenitors of extravillous trophoblasts and syncytiotrophoblast. Reproduction 130:95-103

Kaitu'u-Lino TJ, Brownfoot FC, Beard S, Cannon P, Hastie R, Nguyen TV, Binder NK, Tong S, Hannan NJ (2018) Combining metformin and esomeprazole is additive in reducing sFlt- 1 secretion and decreasing endothelial dysfunction-implications for treating preeclampsia. PLoS One 13:e0188845

Kaitu'u-Lino TJ, Brownfoot FC, Hastie R, Chand A, Cannon P, Deo M, Tuohey L, Whitehead C, Hannan NJ, Tong S (2017) Activating transcription factor 3 is reduced in preeclamptic placentas and negatively regulates sFlt-1 (soluble fms-like tyrosine kinase 1), soluble endoglin, and proinflammatory cytokines in placenta. Hypertension 70:1014-1024

Kim KH, Lee MS (2014) Autophagy—a key player in cellular and body metabolism. Nat Rev Endocrinol 10:322-337

Lamark T, Svenning S, Johansen T (2017) Regulation of selective autophagy: the p62/SQSTM1 paradigm. Essays Biochem 61:609-624

Levine B, Kroemer G (2019) Biological functions of autophagy genes: a disease perspective. Cell 176:11-42

Liu L, Zhuang X, Jiang M, Guan F, Fu Q, Lin J (2017) ANGPTL4 mediates the protective role of PPAR $\gamma$ activators in the pathogenesis of preeclampsia. Cell Death Dis 8:e3054

Malfertheiner P, Kandulski A, Venerito M (2017) Proton-pump inhibitors: understanding the complications and risks. Nat Rev Gastroenterol Hepatol 14:697-710

Mazure NM, Pouysségur J (2010) Hypoxia-induced autophagy: cell death or cell survival? Curr Opin Cell Biol 22:177-180

Mol BWJ, Roberts CT, Thangaratinam S, Magee LA, de Groot CJM, Hofmeyr GJ (2016a) Pre-eclampsia. Lancet (london, England) 387:999-1011

Mol BW, Roberts CT, Thangaratinam S, Magee LA, de Groot CJ, Hofmeyr GJ (2016b) Pre-eclampsia. Lancet 387:999-1011

Nakashima A, Aoki A, Kusabiraki T, Cheng SB, Sharma S, Saito S (2017) Autophagy regulation in preeclampsia: pros and cons. J Reprod Immunol 123:17-23
Nakashima A, Tsuda S, Kusabiraki T, Aoki A, Ushijima A, Shima T, Cheng SB, Sharma S, Saito S (2019) Current understanding of autophagy in pregnancy. Int J Mol Sci 20

Onda K, Tong S, Beard S, Binder N, Muto M, Senadheera SN, Parry L, Dilworth M, Renshall L, Brownfoot F, Hastie R, Tuohey L, Palmer K, Hirano T, Ikawa M, Kaitu'u-Lino T, Hannan NJ (2017) Proton pump inhibitors decrease soluble fms-like tyrosine kinase- 1 and soluble endoglin secretion, decrease hypertension, and rescue endothelial dysfunction. Hypertension 69:457-468

Phipps EA, Thadhani R, Benzing T, Karumanchi SA (2019) Preeclampsia: pathogenesis, novel diagnostics and therapies. Nat Rev Nephrol 15:275-289

Rana S, Lemoine E, Granger JP, Karumanchi SA (2019) Preeclampsia: pathophysiology, challenges, and perspectives. Circ Res 124:1094-1112

Ravanan P, Srikumar IF, Talwar P (2017) Autophagy: the spotlight for cellular stress responses. Life Sci 188:53-67

Saleh L, Samantar R, Garrelds IM, van den Meiracker AH, Visser W, Danser AHJ (2017) Low soluble Fms-like tyrosine kinase-1, endoglin, and endothelin-1 levels in women with confirmed or suspected preeclampsia using proton pump inhibitors. Hypertension 70:594-600

Sandrim VC, Caldeira-Dias M, Montenegro MF (2019) Esomeprazole to treat women with preeclampsia: possible implications in the nitric oxide homeostasis. Am J Obstet Gynecol 220:206-207

Sasaki Y, Darmochwal-Kolarz D, Suzuki D, Sakai M, Ito M, Shima T, Shiozaki A, Rolinski J, Saito S (2007) Proportion of peripheral blood and decidual CD4(+) CD25(bright) regulatory T cells in pre-eclampsia. Clin Exp Immunol 149:139-145

Tache V, Ciric A, Moretto-Zita M, Li Y, Peng J, Maltepe E, Milstone DS, Parast MM (2013) Hypoxia and trophoblast differentiation: a key role for PPAR $\gamma$. Stem Cells Dev 22:2815-2824

Tomimatsu T, Mimura K, Matsuzaki S, Endo M, Kumasawa K, Kimura T (2019) Preeclampsia: maternal systemic vascular disorder caused by generalized endothelial dysfunction due to placental antiangiogenic factors. Int J Mol Sci 20

Tong S, Kaitu'u-Lino TJ, Onda K, Beard S, Hastie R, Binder NK, Cluver C, Tuohey L, Whitehead C, Brownfoot F, De Silva M, Hannan NJ (2015) Heme oxygenase-1 is not decreased in preeclamptic placenta and does not negatively regulate placental soluble fms-like tyrosine kinase-1 or soluble endoglin secretion. Hypertension 66:1073-1081

Xu J, Ji J, Yan XH (2012) Cross-talk between AMPK and mTOR in regulating energy balance. Crit Rev Food Sci Nutr 52:373-381

Yamanaka-Tatematsu M, Nakashima A, Fujita N, Shima T, Yoshimori T, Saito S (2013) Autophagy induced by HIF1 $\alpha$ overexpression supports trophoblast invasion by supplying cellular energy. PLoS One 8:e76605

Zhang Y, Jiang G, Zhang C (2021) Downregulation of Cullin 3 ligase signaling pathways contributes to hypertension in preeclampsia. Front Cardiovasc Med 8:654254

Publisher's Note Springer Nature remains neutral with regard to jurisdictional claims in published maps and institutional affiliations. 